\title{
Gold nanowires from silicon nanowire templates
}

\author{
T. C. Wong, C. P. Li, R. Q. Zhang, ${ }^{\text {a) }}$ and S. T. Lee ${ }^{\text {b) }}$ \\ Center of Super-Diamond and Advanced Films (COSDAF) and Department of Physics and Materials \\ Science, City University of Hong Kong, Hong Kong SAR, China
}

(Received 25 August 2003; accepted 19 November 2003)

\begin{abstract}
We report a simple method for producing gold nanowires (AuNWs) by using silicon nanowires (SiNWs) as templates. Uniform AuNWs were formed in the core of SiNWs, when SiNWs coated with $\mathrm{Au}$ were furnace annealed at $\sim 880^{\circ} \mathrm{C}$ at $10^{-2}$ Torr. Transmission electron microscopy (TEM) examination showed the AuNWs had diameters of $\sim 10 \mathrm{~nm}$. High-resolution TEM revealed lattice fringes with an interlayer spacing of $0.235 \mathrm{~nm}$, which corresponds to the spacing in Au crystal, confirming the AuNWs are crystalline. (C) 2004 American Institute of Physics.
\end{abstract}

[DOI: $10.1063 / 1.1641179$ ]

In recent years, one-dimensional nanostructured materials have attracted increasing interest. Semiconductor nanowires are one of the focuses of intensive research because of their potential applications in electronic, photonic, and optoelectronic devices. ${ }^{1-3} \mathrm{Au}$ nanowires (AuNWs) are also attracting wide attention, ${ }^{4-9}$ as $\mathrm{Au}$ is well-known as an outstanding interconnect because of its good electrical conductivity and stability in an oxidizing environment. Various techniques including electrochemical fabrication, ${ }^{4-6}$ scanning tunneling microscope (STM), ${ }^{7}$ and oblique evaporation of metal on prestructured substrates ${ }^{8}$ have been developed for synthesizing AuNWs. Among the existing methods to produce AuNWs, the STM method is time consuming and thus not suitable for large-scale production, and metal evaporation can only produce nanowires in small quantities. ${ }^{9}$ Here, we present a simple furnace method to synthesize AuNWs by heating silicon nanowires (SiNWs) as a template. Compared with previous methods, the present synthesis is easy and simple. In addition, large-scale production of AuNWs could be fabricated with this method without complicated preparation.

SiNWs were prepared by thermal decomposition of pure $\mathrm{SiO}$ powders (Aldrich, 325 mesh, 99.9\%) in a quartz tube in a tube furnace. The chamber was heated to $1200^{\circ} \mathrm{C}$ under a carrier gas $\left(5 \% \mathrm{H}_{2}, 95 \% \mathrm{Ar}\right)$ at $50 \mathrm{sccm}$ flow rate. The light-brown SiNWs were grown inside the wall and had a diameter of several nanometers to tens of nanometers. ${ }^{10}$ SiNWs used in this study were $\sim 20 \mathrm{~nm}$ in diameter and covered with an oxide layer. The as-grown SiNWs were etched with $5 \%$ hydrogen fluoride solution to remove the oxide layer, and rinsed with de-ionized water. The processed SiNWs were then placed on the carbon-coated copper grids. Gold deposition was carried out by using argon ion sputtering (Fine Coat JFC-1100E) on both sides of the sample for $20 \mathrm{~s}$ at room temperature at a pressure of $10^{-2}$ Torr. The Au-coated samples were placed inside the quartz tube in the middle of the furnace, and were heated up in two different stages with a heating rate of $40{ }^{\circ} \mathrm{C} / \mathrm{min}$ : (1) from room temperature $\left(25^{\circ} \mathrm{C}\right)$ to $500^{\circ} \mathrm{C}$ and (2) from room temperature

\footnotetext{
${ }^{\text {a)} E l e c t r o n i c ~ m a i l: ~ a p r q z @ ~ c i t y u . e d u . h k ~}$

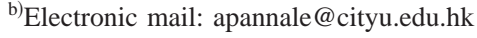

$\left(25^{\circ} \mathrm{C}\right)$ to $880^{\circ} \mathrm{C}$. The annealing of the samples lasted for 1 $\mathrm{h}$ under a pressure of $10^{-2}$ Torr without a carrier gas. The annealed samples were examined by a high-resolution transmission electron microscope (HRTEM) (Philips CM200 FEG) operated at $200 \mathrm{kV}$.

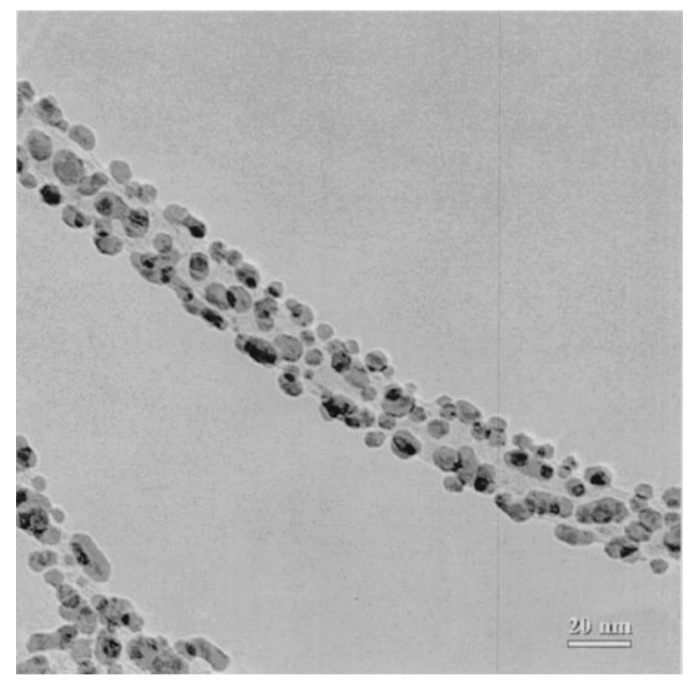

(a)

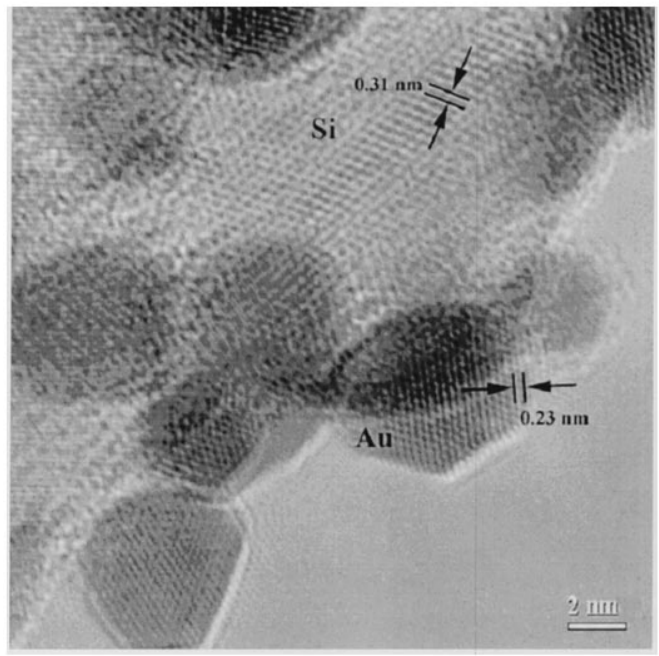

(b)

FIG. 1. (a) TEM image of SiNWs coated with Au nanoparticles, and (b) the corresponding HRTEM image. 


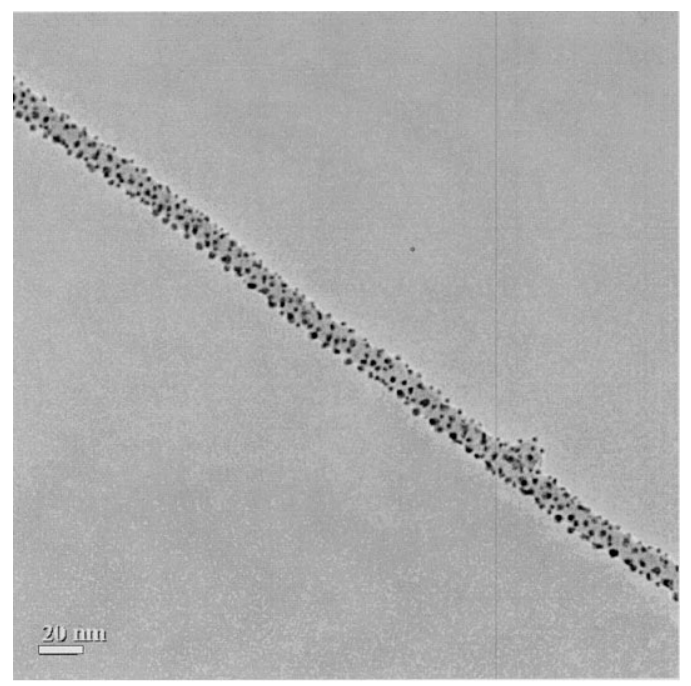

(a)

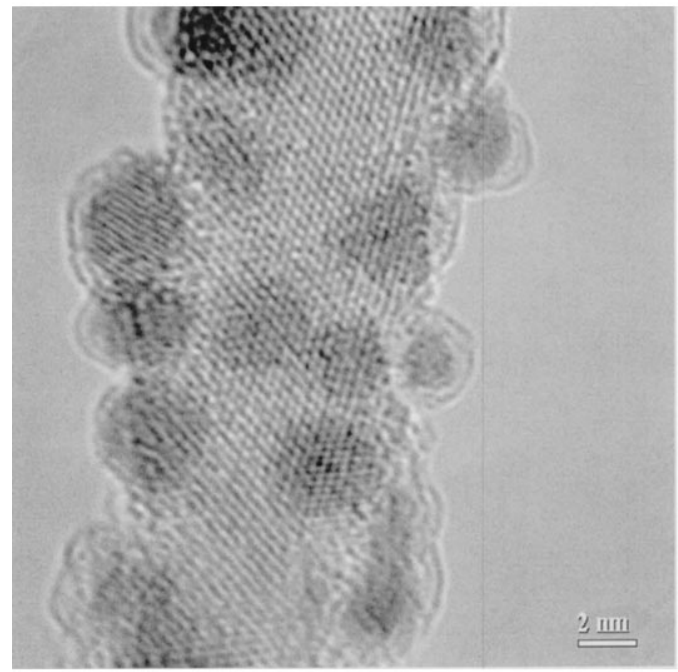

(b)

FIG. 2. (a) TEM image of Au nanoparticles attached on the surface of SiNWs after annealing at $500^{\circ} \mathrm{C}$ for $1 \mathrm{~h}$ at $10^{-2}$ Torr, and (b) the corresponding HRTEM image.

Figure 1(a) shows the SiNWs coated with Au nanoparticles after ion sputtering. It is clear that $\mathrm{Au}$ nanoparticles were randomly distributed on the whole surface of the SiNW. The nanoparticles have an average diameter of $5 \mathrm{~nm}$, and are closely attached on the surface of SiNWs. Figure 1(b) is a HRTEM image of SiNWs coated with Au nanoparticles. From the HRTEM examination, the lattice parameter $(d$ spacing) of the crystalline silicon core was found to have a lattice spacing of $0.31 \mathrm{~nm}$, while the Au nanoparticles had a lattice spacing of $0.23 \mathrm{~nm}$, which corresponds to the [111] planes of Au crystal. No gold silicide was found, in contrast to the formation of gold silicide under argon ion sputtering of Au on silicon. ${ }^{10}$

Upon furnace annealing at $500{ }^{\circ} \mathrm{C}$ and $10^{-2}$ Torr for $1 \mathrm{~h}$, the Au nanoparticles remained randomly attached on the surface of SiNWs, as shown in Fig. 2(a). The size of the Au nanoparticles and SiNWs underwent little change. The HRTEM image in Fig. 2(b) shows that crystalline silicon was still present inside the core of the nanowire, but the nanoparticles changed shape and moved towards the core of the nanowires. Some of the nanoparticles even exhibited a Downloaded 21 Apr 2010 to 150.203.243.34. Redistribution subject

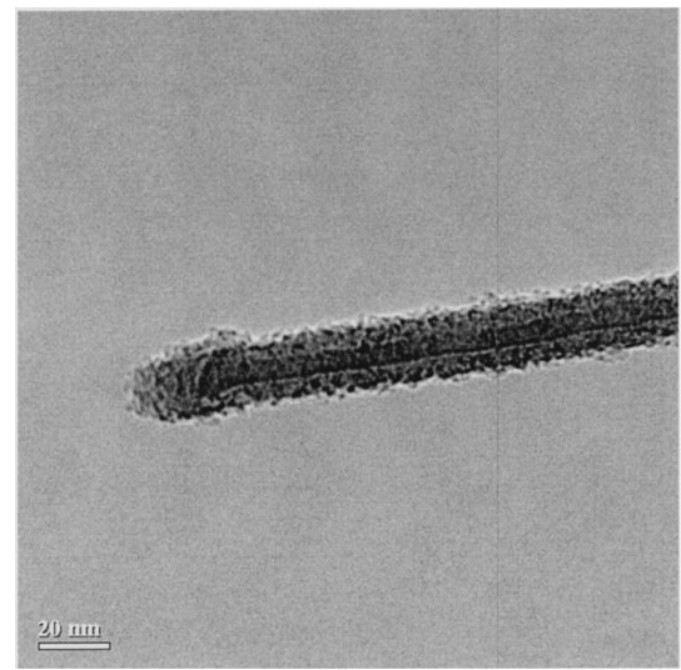

(a)

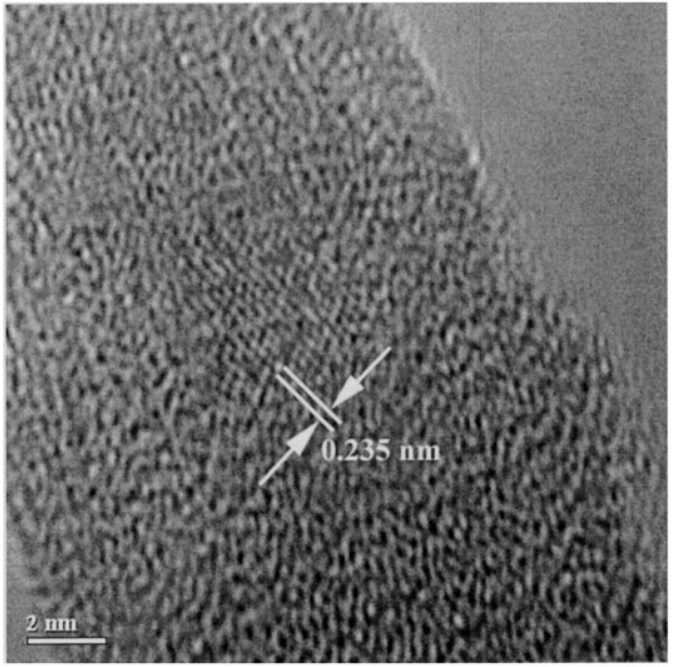

(b)

FIG. 3. (a) TEM image of AuNW inside a $\mathrm{SiO}_{x}$ sheath formed by the annealing of the Au-coated SiNW at $880^{\circ} \mathrm{C}$ for $1 \mathrm{~h}$ at $10^{-2}$ Torr, and (b) the corresponding HRTEM image showing the Au core.

highly oriented relationship with the SiNW core as shown in Fig. 2(b). Upon annealing at $880^{\circ} \mathrm{C}$ and $10^{-2}$ Torr for $1 \mathrm{~h}$, no $\mathrm{Au}$ nanoparticles were found attached on the surface of the SiNW, as shown in Fig. 3(a). In the core of the wire, a long, thin nanowire with a darker contrast was formed. The HRTEM image shown in Fig. 3(b) reveals a lattice spacing of $0.235 \mathrm{~nm}$, revealing the formation of AuNWs sheathed by amorphous silicon oxide. The average diameter of the $\mathrm{Au}$ nanowire is about $10 \mathrm{~nm}$ in the core.

The formation mechanism of the AuNWs is illustrated schematically in Fig. 4. First, the deposited Au nanoparticles attached on the surface of a SiNW [Fig. 4(a)]. Upon heating the coated SiNWs, shown in Fig. 4(b), some of the surface hydrogen atoms started desorbing ${ }^{11}$ and a relatively soft matrix for the Au nanoparticles was formed, accompanied with surface oxidization. The Au nanoparticles tended to move into the core associated with the motion of the $\mathrm{Si}-\mathrm{SiO}_{x}$ interface of the wire so as to reduce the surface area and thus the surface energy. Heating to $880^{\circ} \mathrm{C}$, the Au nanoparticles would be in a semiliquid state ${ }^{12}$ as the annealing temperature was slightly below the melting point of $\mathrm{Au} .{ }^{13}$ Meanwhile, the surface hydrogen atoms were totally evaporated and the core to AIP license or copyright; see http://apl.aip.org/apl/copyright.jsp 
(a)

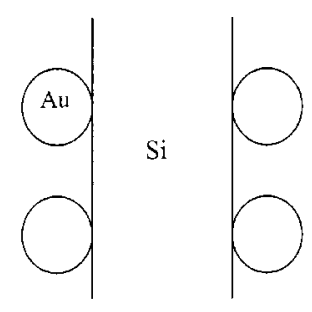

(b)
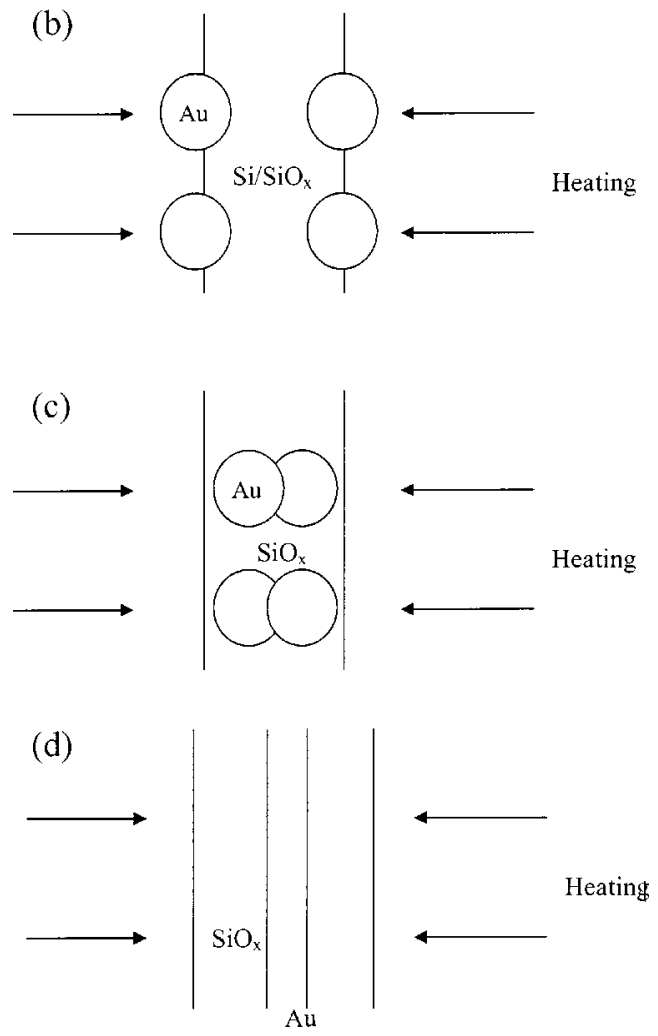

FIG. 4. Schematic diagrams showing the formation mechanism of AuNWs by furnace annealing: (a) Au nanoparticles deposited by argon ion sputtering are attached on the surface of a SiNW, (b) oxidation of SiNWs and diffusion of Au nanoparticles into SiNWs under heating, (c) diffusion of Au nanoparticles in the $\mathrm{SiO}_{x}$ matrix of the nanowire at elevated temperature (e.g., $880^{\circ} \mathrm{C}$ ), and (d) formation of $\mathrm{AuNW}$ in the $\mathrm{SiO}_{x}$ matrix.

of SiNWs gradually oxidized into silicon suboxide $\left(\mathrm{SiO}_{x}\right)$, as shown in Fig. 4(c). The crystalline structure of silicon was changed to an amorphous structure, which is soft enough allowing diffusion of Au nanoparticles into the core of nanowire. It is expected that a higher annealing temperature would facilitate faster nanoparticle diffusion toward the core of nanowire.

There are two possible reactions that could occur inside the core of SiNWs. First, Au nanoparticles may react with silicon to form gold silicide $\left(\mathrm{Au}_{x} \mathrm{Si}_{y}\right)$. Second, the nanoparticles may precipitate together to reduce their surface energy. For the first one, the suitable temperature is between 370 and
$500{ }^{\circ} \mathrm{C} .{ }^{14,15}$ Considering the annealing temperature in our case is much higher than this temperature range, the second possibility is most likely the case. In fact, we did not find any gold silicides inside the nanowire under HRTEM examination. As a result, $\mathrm{Au}$ nanoparticles condense together and form the interface between $\mathrm{Au}$ and $\mathrm{Si}$, leading to the formation of AuNWs, as illustrated in Fig. 4(d).

The surface composition of SiNWs and the size of the nanoparticles were found to affect the AuNWs formation. Under a similar treatment condition, the as-grown, oxidecoated SiNWs (no HF treatment) underwent no structural change after Au deposition and annealing. Annealing time is also important for AuNWs formation, as it takes time for the $\mathrm{Au}$ nanoparticles to diffuse and precipitate in the core of nanowires.

In summary, furnace annealing of Au-coated SiNWs has been used to synthesize crystalline AuNWs embedded in a $\mathrm{SiO}_{x}$ sheath. The formation of AuNWs is due to the softening of SiNWs upon oxidation and the enhanced Au diffusion at elevated temperatures. The $\mathrm{SiO}_{x}$ overlayer of AuNWs can be readily removed by $\mathrm{HF}$ etching to expose the AuNWs, if necessary. Thus, the furnace annealing of Au-coated SiNWs offers a simple method of synthesizing thin and largequantity crystalline Au nanowires. The synthesized AuNWs should find interesting applications in nanodevices.

The work described in this letter is supported by two grants from the Research Grants Council of the Hong Kong SAR, China (Project No. 8730016, e.g., CityU 3/01C; Project No. 9040633, e.g., CityU 1011/01P), and by a grant from the Chinese Academy of Sciences, China.

${ }^{1}$ S. Iijima, Nature (London) 354, 56 (1991).

${ }^{2}$ H. Dai, E. W. Wong, Y. Z. Lu, S. Fan, and C. M. Lieber, Nature (London) 375, 769 (1995).

${ }^{3}$ A. P. Alivisatos, Science 271, 933 (1996).

${ }^{4}$ C. Ji and Peter C. Searson, Appl. Phys. Lett. 81, 4437 (2002).

${ }^{5}$ P. Forrer, F. Schlottig, H. Siegenthaler, and M. Textor, J. Appl. Electrochem. 30, 533 (2000).

${ }^{6}$ X. Y. Zhang, L. D. Zhang, Y. Lei, L. X. Zhao, and Y. Q. Mao, J. Mater. Chem. 11, 1732 (2001).

${ }^{7}$ H. C. Manoharan, C. P. Lutz, and D. M. Eigler, Nature (London) 403, 512 (2000).

${ }^{8}$ D. F. Prober, M. D. Feuer, and N. Giordaro, Appl. Phys. Lett. 37, 94 (1980).

${ }^{9}$ J. Jorritsma, M. A. M. Gijs, C. Schonenberger, and G. H. Stiener, Appl. Phys. Lett. 64, 1489 (1995).

${ }^{10}$ S. T. Lee, Y. F. Zhang, N. Wang, Y. H. Tang, I. Bello, C. S. Lee, and Y. W. Chung, J. Mater. Res. 12, 4503 (1999).

${ }^{11}$ X. H. Sun, S. D. Wang, N. B. Wong, D. D. D. Ma, and S. T. Lee, Inorg. Chem. 42, 2398 (2003).

${ }^{12}$ Y. G. Wang, A. Z. Jin, and Z. Zhang, Appl. Phys. Lett. 81, 4425 (2002).

${ }^{13}$ W. D. Callister, Jr., Materials Science and Engineering, An Introduction, 5th ed. (Wiley, New York, 2000).

${ }^{14}$ J. T. Hu, T. W. Odom, and C. M. Lieber, Acc. Chem. Res. 32, 435 (1999).

${ }^{15}$ K. K. Lew, C. Reuther, A. H. Carim, and J. M. Redwing, J. Vac. Sci. Technol. B 20, 390 (2002). 